\title{
Multiwavelength observations of GRB afterglows
}

\author{
Alberto J. Castro-Tirado \\ Instituto de Astrofísica de Andalucía (IAA-CSIC), Glorieta de las Astronomía s/n, E-18080 \\ Granada, Spain \\ email: ajct@iaa.es
}

\begin{abstract}
Multiwavelength observations of gamma-ray burst afterglows are presented, in particular those in the optical and millimetre wavelengths. I will focus on the observations mostly carried out at Spanish ground-based observatories (mainly the $10.4 \mathrm{~m}$ GTC) and at the Plateau de Bure Interferometer in the French Alps. The importance of global networks of robotic telescopes (like BOOTES, established worldwide) for early time observations in order to put constraints on the physical mechanisms of the GRB early time emission phase is also discussed. The overall observational efforts provide additional clues for a better understanding of the reverse and forward shock. Finally I will report on the Lomonosov/UFFO-p capabilities taking into account its launch in 2012.
\end{abstract}

Keywords. gamma-rays: bursts, accretion disks, acceleration of particles, black hole physics

\section{Introduction}

Since their discovery in gamma-rays in 1967 and the detection of the first counterparts at other wavelengths in 1997, thanks to the BeppoSax improvement on the localization accuracy leading to the first X-ray afterglow discovery (Costa et al. 1997), now we know that Gamma-Ray Bursts (GRBs) originate at cosmological distances with energy releases of $10^{51}-10^{53}$ ergs. The multiwavelength emission that follows the gamma-ray emission (the "afterglow") satisfies the predictions of the "standard" relativistic fireball model. See Castro-Tirado (2001) for a review of the field prior to the year 2000.

Since the launch of the Swift mission in 2004, more than 500 GRBs have been detected, with $85 \%$ of them being recorded in follow-up X-ray observations, $60 \%$ with optical detection and nearly 200 redshifts have been measured since 1997 (including 41 prior to Swift). Fermi was launched in 2008, to record the higher energy (MeV) population.

For the record, it is worth mention that a likely X-ray afterglow was pinpointed $5 \mathrm{yr}$ before the BeppoSAX detection of GRB 970228. Such was the case for GRB 920723B (Castro-Tirado 1994), and the word "afterglow" was indeed used by Terekhov et al. (1993). Moreover, the first optical afterglow was already serendipitously imaged in 1992 for GRB 920925c (Hurley et al. 2000) being reported 15 yr later (Denisenko \& Terekhov 2007).

\section{Afterglow science in long-duration GRBs}

Since the initial works by Paczynski (1986) and Goodman (1986), the physics of relativistic ejections by a compact source was further developed. The standard model was developed by Meszaros \& Rees (1997) and also by Paczynski \& Rhoads (1993). Hereafter, we will consider a few selected results on both the reverse and forward shock emission. 

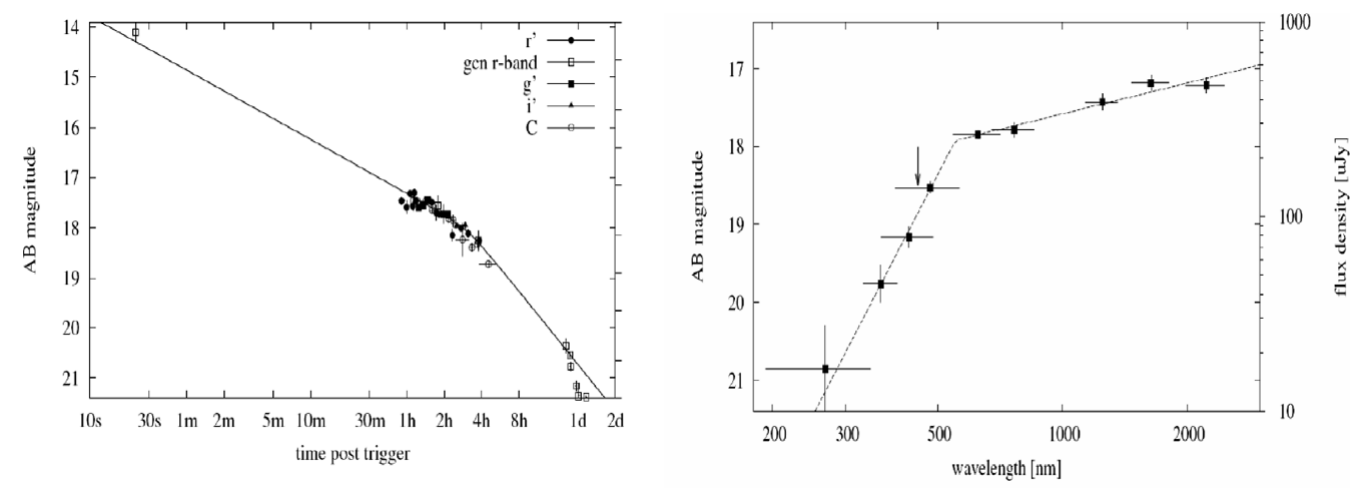

Figure 1. Left panel: The overall lightcurve of the GRB 080603B afterglow including BOOTES data. Right panel: The spectral energy distribution of the optical afterglow in the rest frame. The small arrow marks the Ly- $\alpha$ position for $\mathrm{z}=2.69$. From Jelinek et al. (2012).

\subsection{The reverse shock emission}

Due to the interaction with the surrounding medium, a reverse shock propagates within the ejecta. It is usually believed to contribute to the prompt emission (optical flash) and/or the early afterglow. For a uniform shell, the reverse shock is short-lived.

An important assumption is that the reverse shock microphysics parameters are equivalent to the internal shock microphysics parameters and different (in both cases) to the forward shock ones. If the outflow is variable, the reverse shock can be much more complicated than this simple picture and can even be long-lived. Thus, the strength of the reverse shock depends on magnetization content of the ejecta (Zhang, Kobayashi \& Meszaros 2003, Gomboc et al. 2009).

For instance, for the case of GRB 060117 (Jelinek et al. 2006) and GRB 090902B (Pandey et al. 2010), a bright afterglow and a decay slope are suggestive of a reverse shock origin.

\subsection{The forward shock emission}

The afterglow is usually interpreted as the signature of the deceleration of the relativistic outflow by the external medium. The forward shock can be modelled under the frame of the standard fireball model, by means of the synchrotron spectrum moving towards longer wavelengths as function of time.

In the case of GRB 060117 (Jelinek et al. 2006), the peak time of the rising optical afterglow lightcurves could be used for determining the initial Lorentz factor following Molinari et al. (2007). The rising lightcurves $\left(F \propto t^{\alpha}\right)$ are also important to understand the onset of the afterglow (Sari et al. 1999): $\alpha \sim 2\left(\nu_{c}<\nu_{\text {optical }}\right)$ or $\alpha \sim 3\left(\nu_{c}<\nu_{\text {optical }}\right)$ in the case of ISM or $\alpha \sim 0.5$ for a WIND density profile. It allows also off-axis and structured jet models to be constrained (Panaitescu et al. 1998).

Prior to Swift, there were very promising results (multiwavelength fits) but even then problems were noticed, like the jet breaks, first detected in GRB 990123 (Castro-Tirado et al. 1999, Kulkarni et al. 1999; see Fig. 1).

After the initial Swift results, the picture has turned out to be more complicated: the canonical Swift X-ray afterglow lightcurve presents five distinct regions (Nousek et al. 2005): i) a steep decline; ii) a shallow slope; iii) the classical afterglow; iv) a jet break/late plateau; and v) flares (mostly in X-rays).

In many occasions, energy injections have to be taken into account to properly fit $F(\lambda, \mathrm{t})$. Thus, GRB $021004(\mathrm{z}=2.33)$ was modelled by multiple energy injections (de 
Ugarte Postigo et al. 2005), as was GRB $050730(\mathrm{z}=3.97)$ and GRB 051028 (unknown$\mathrm{z})$ (Castro-Tirado et al. 2006). GRB 030329/SN 2003dh ( $\mathrm{z}=0.168$ ) is also modelled by multiple energy injections but the initial phase cannot be properly accounted for (Guziy et al. 2012). GRB 060904B is an example of a GRB displaying all features on its lightcurve (Jelinek et al. 2012).

\subsection{The GRB-highly energetic SN connection}

The first hint for the long duration GRB - highly energetic supernova (SN) connection occurred in 1998, when GRB 980425 took place in the same region of the sky (also coincident in time) with SN 1998bw (Galama et al. 1998, Pian et al. 1999). It exhibited all the characteristics of a core-collapse SN Ic, expect for its kinetic energy $\left(\sim 10^{52} \mathrm{erg}\right)$ and its high luminosity (Iwamoto et al. 1998), with an inferred progenitor mass of $\sim 30$ $\mathrm{M}_{\odot}$ and a remnant mass consistent with that of a black hole. Later on, a rebrightening seen in GRB 980326 was attributed to an underlying SN (Castro-Tirado \& Gorosabel 1999, Bloom et al. 1999) being properly modelled by the contribution of a SN at $z \sim 1$ (Bloom et al. 1999).

Indeed, the smoking gun took place in 2003, when a multiwavelength campaign for the nearby GRB 030329 afterglow (at $\mathrm{z}=0.168$ ) led to the detection of prominent broad emission lines similar to the ones seen in GRB 980425/SN 1998bw (Stanek et al. 2003, Hjorth et al. 2003). The optical afterglow spectrum showed some evolution starting from the first night after the burst, and the beginning of spectral changes were seen as early as $\sim 10-12 \mathrm{~h}$ after the GRB. The onset of the spectral changes for $\mathrm{t}<1$ day indicated that the contribution from a Type Ic supernova (SN) -dubbed SN 2003dh later on- into the optical afterglow flux could be detected much earlier (Sokolov et al. 2003).

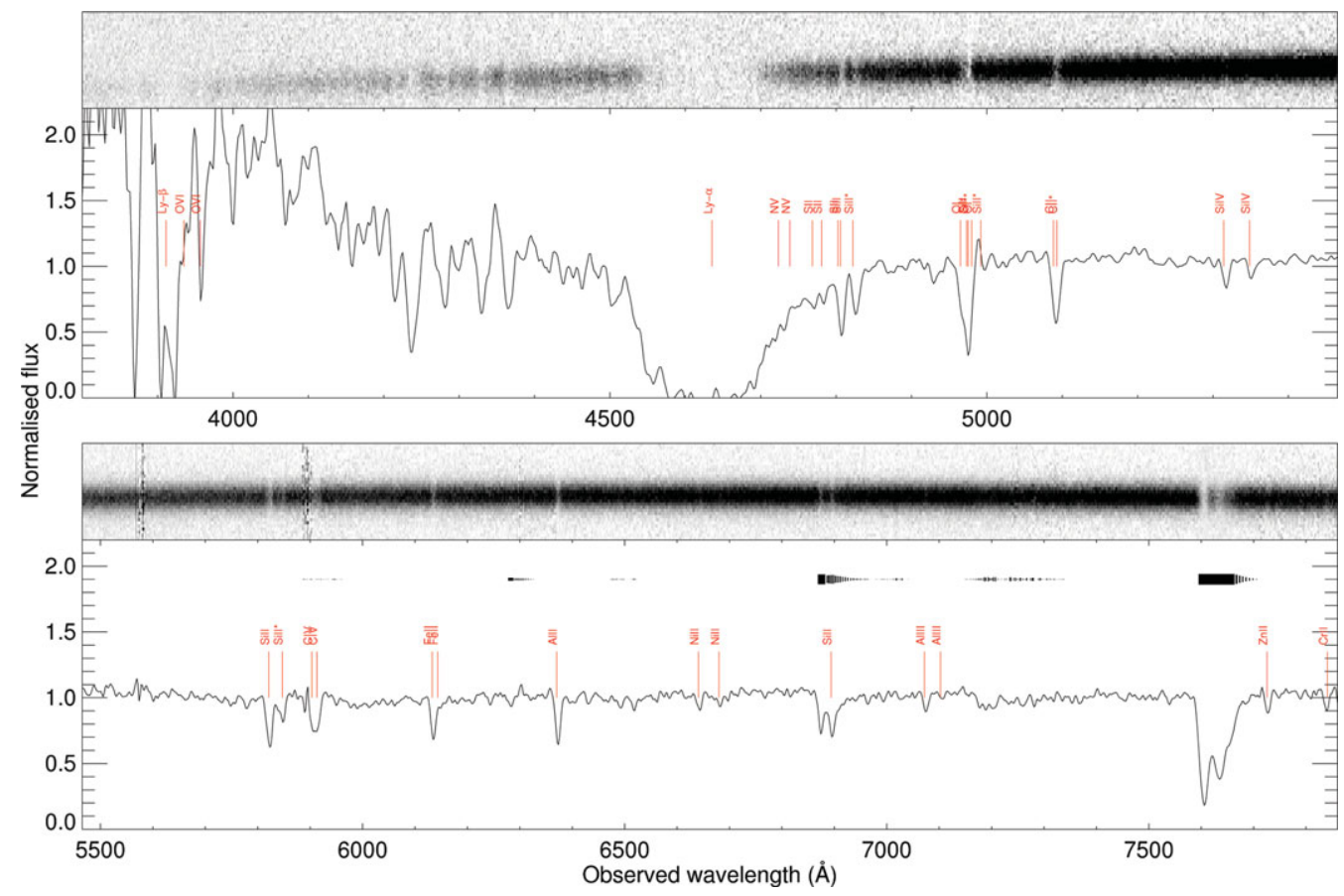

Figure 2. The GTC spectrum of GRB 100327A obtained $~ 2.33 \mathrm{hr}$ after the trigger with the $10.4 \mathrm{~m}$ GTC telescope (+OSIRIS). A strong DLA system (with $\mathrm{N}_{\mathrm{H}} \sim 10^{22} \mathrm{~cm}^{-2}$ is detected, besides several absorption lines, at a common redshift of 2.813. From Castro-Tirado et al. (2012c). 
Rebrightenings have been observed for many long-duration GRBs at epochs consistent with a rising light curve from a SN exploding within 1 day of the GRB time, a few of them being confirmed by spectroscopy and changes of colours around $\sim 15(1+z)$ days after the GRB trigger. Thus, SNe/GRB should be reachable with the Spanish $10.4 \mathrm{~m}$ GTC up to $\mathrm{z} \sim 1$. In fact, GTC has allowed detection of the underlying SNe for both GRB 091127 (SN 2009nz) at $\mathrm{z}=0.490$ (Vergani et al. 2011a; see also Cobb et al. 2010, Berger et al. 2011) and GRB 111211A (de Ugarte Postigo et al. 2012).

\subsection{Spectroscopic observations of GRB afterglows}

Besides helping to determine the distance scale, spectroscopic observations of nearby events $(\mathrm{z}<0.3)$ may reveal on a few cases unequivocal SN signatures similar to those of SN 1998bw, which coupled to the lightcurve monitoring will help to estimate the energy output, ${ }^{56} \mathrm{Ni}$ mass and remnant mass of the associated SN.

Besides this, optical (and near-IR) spectroscopy is most essential for understanding the GRB environment (abundances, metallicities, etc). As an example, in GRB 021004 ( $\mathrm{z}=$ 2.33 ), high resolution spectroscopy revealed several high velocity systems in the range 200-3000 km/s (Starling et al. 2005, Castro-Tirado et al. 2010, Vergani et al. 2011b). In GRB 021004, the high velocity systems were naturally explained by multiple shells formed by stellar winds of a WR progenitor after passing through a LBV phase after reaching the Eddington limit. $\mathrm{A} \sim 60 \mathrm{M}_{\odot}$ ZAMS progenitor is suggested following the accepted evolutionary track: O - LBV - WR - SN (Castro-Tirado et al. 2010).

We have also used the $10.4 \mathrm{~m}$ GTC (+OSIRIS) to determine redshifts for a handful of events (GRB 100316A, 110503, 110801A, 110918A and 120326A; Sánchez-Ramírez et al. 2012, Tello-Salas et al. 2012) and confirmed many others (like GRB 100816A, 110422A, 110918A and 120327A; see Fig. 2).

\subsection{Polarimetric observations of GRB afterglows and hypernovae}

Optical light of the afterglows should be polarized because the magnetic field of the ISM, and also the central source, are amplified in the shocked front, so the electrons emit polarized synchrotron light. The ejecta concentrates the radiation in a beam of width $\theta$ $\sim 1 / \Gamma(\mathrm{t})$, where $\Gamma(\mathrm{t})$ is the Lorentz factor. The observer line of sight must be within the beam (otherwise the GRB would be undetectable) but in general not perfectly aligned with the jet. This breaks the source-observer axi-symmetry, avoiding the cancellation of electric vectors created in different emitting regions (Lazzati et al. 2004).

Observations performed so far show varying levels of polarized emission in the jet and the geometry. Polarized optical emission has been claimed for a few classical GRBs, usually $\mathrm{P}=1-2 \%$ (Gorosabel et al. 2004) and rarely up to $10 \%$ (Steele et al. 2009). P $1.5 \%$ was measured for SN 2003dh / GRB 030329 (Greiner et al. 2003).



Figure 3. Left panel: NGC 2770 in broad-band optical filters showing the location of SN2007uy and SN 2008D. Right panel: The same field imaged in H- $\alpha$ thanks to the OSIRIS tunable filters in the GTC. From Gorosabel et al. (2011). 



Figure 4. Left panel: the GRB $051022 \mathrm{~mm}$ afterglow was coincident with a blue galaxy at redshift $\mathrm{z}=0.809$. Right panel: the SED of the dark GRB 051022 afterglow. From Castro-Tirado et al. (2010).

The optical light of hypernovae can also be polarized as in the case of normal supernovae: Thomson scattering in the expanding stellar atmosphere. If the expansion is perfectly spherical, the polarization coming from different stellar parts would be canceled so no net polarization would be detected, as in normal SNe. However, in hypernovae, the expansion is likely to be ellipsoidal, so the symmetry around the line of sight is broken, producing non-zero polarization.

For SN 2006aj (type Ic) / XRF 060218A there was evidence for aspherical expansion with $\mathrm{P}=4 \%$ decreasing to $1.4 \%$ plus a $100^{\circ}$ rotation in the position angle (Gorosabel et al. 2006). For SN 2008D / XRT 080109 in NGC 2270 there was evidence for aspherical expansion with $\mathrm{P}=1 \%$ (Gorosabel et al. 2010). Neither SN 2008D nor XRT 080109 are associated with any given giant H-II region in NGC 2770 (Fig. 3).

\subsection{Afterglows at $m m$ wavelengths}

Millimetre observations are not affected by high-z or extinction and usually can lead to sampling the synchrotron peak in the spectrum as well as detecting the tail of the prompt emission and the forward shock. Since the first mm afterglow ever detected by us (Bremer et al. 1998), the number of follow-ups amounted to 60, with 54 events being observed at $3 \mathrm{~mm}$ and 20 of them being detected so far. Redshifts for the GRB afterglows lie in the range $\mathrm{z}=0.03-9.2$, with measured flux densities (at $3 \mathrm{~mm}$ ) varying between 0.25 and $60 \mathrm{mJy}$ (but usually $<1.5 \mathrm{mJy}$ ) with the first observations taking place around 1-2 days after the GRB. We have detected 2 (out of 3) GRBs at $\mathrm{z}>6$ : GRB $050904(\mathrm{z}=$ 6.3) and GRB $090423(\mathrm{z}=8.3)$. On the lower redshift end, we have detected the low-z, faint GRB 080109 / SN 2008D ( $\mathrm{z}=0.0065)$. Amongst the long-duration class, we have detected two "dark" GRBs: one associated with a $\mathrm{z}=0.809$ galaxy (GRB 051022, Fig. 4) and another one (GRB 090404) whose host galaxy is not properly identified. Altogether, this implies a $\sim 42 \%$ success rate (for this non-blind sample). A more in-depth analysis is be presented in Castro-Tirado et al. (2012a).

\section{Automated and Robotic Telescopes and their usage for GRB follow-ups}

The (B)urst (O)bserver and (O)ptical (T)ransient (E)xploring (S)ystem (BOOTES), is a set of instruments that was conceived in 1995 and nowadays comprises four astronomical 

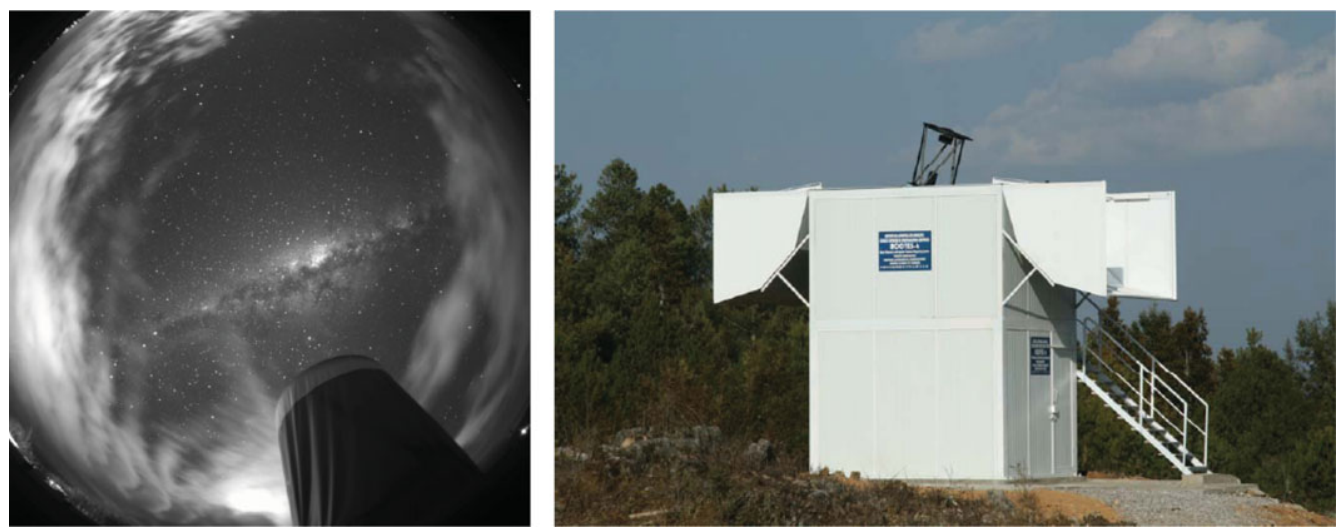

Figure 5. Left panel: an image obtained by the CASANDRA-3 all-sky camera showing the silhouette of the $0.6 \mathrm{~m}$ diameter YA telescope against the Milky Way at the BOOTES-3 robotic astronomical station in Blenheim (New Zealand). Right panel: the enclosure of $0.6 \mathrm{~m}$ diameter MET telescope at the BOOTES-4 robotic astronomical station in Lijiang (China).

stations in Spain (two), New Zealand and China (Fig. 5). The main scientific goal is the follow-up of GRBs by means of fast ultralight weight $60 \mathrm{~cm}$ diameter telescopes (CastroTirado et al. 2012b).

BOOTES-1 follow-ups have been partially summarized by Jelinek et al. (2011). The BOOTES network has allowed the discovery of a handful of optical afterglows (GRB 080330, GRB 080603B, GRB 080605, GRB 080606, GRB 101112A), observing many other at early times. BOOTES-2 and BOOTES-3, with larger diameter telescopes, have also contributed to some detailed studies on the forward shock evolution, as in the case of GRB 080603B, GRB 080605 and GRB 080606 (Jelinek et al. 2012) including the discovery of the optical afterglows of GRB 091029 and GRB 091208B. On the aggregate, BOOTES-1 have followed $\sim 40$ events, detecting 10 afterglows, BOOTES-2/TELMA have followed up 9 events, detecting 3 afterglows, whereas BOOTES-3/YA have followed up 17 events, detecting 5 afterglows. BOOTES-4/MET has just followed up two GRBs, as it was officially opened in Mar 2012.

\section{UFFO-p onboard Lomonosov}

GRB afterglows can also be monitored by doing the follow-up using the triggering satellite itself, besides sending the position to the Earth (BeppoSAX in 6-8 hr, Swift in 1 min). Early follow-up (within $\sim 1 \mathrm{hr}$ ) is only available to Swift so far (sometimes even very early, with response times of $\sim 1 \mathrm{~min}$ ) due to the slewing time of the entire spacecraft. Is it possible to beat this 1 min barrier from space? Indeed, in UFFO-p (UFFO-pathfinder) the optical path moves, not the spacecraft itself. This is achieved thanks to a fast slewing motorized mirror (new concept) in the Slewing Mirror Telescope (SMT) instrument (a mirror of $10 \mathrm{~cm}$ diameter) and an intensified CCD at the Cassegrain focus to achieve less than $1 \mathrm{~s}$ optical response after X-ray trigger. The second instrument is the UFFO Burst Alert \& Trigger telescope (UBAT) for detecting the GRB locations. The UBAT employs a coded-mask $\gamma /$ X-ray camera with a wide field of field of $\sim 1.8$ sterad $\left(90^{\circ} \times 90^{\circ}\right)$ and is comprised of three parts: a coded mask, a hopper, and a detector module. The UBAT DM consists of a LYSO scintillator crystal array sensitive to $\sim 200 \mathrm{keV}$ (effective area 

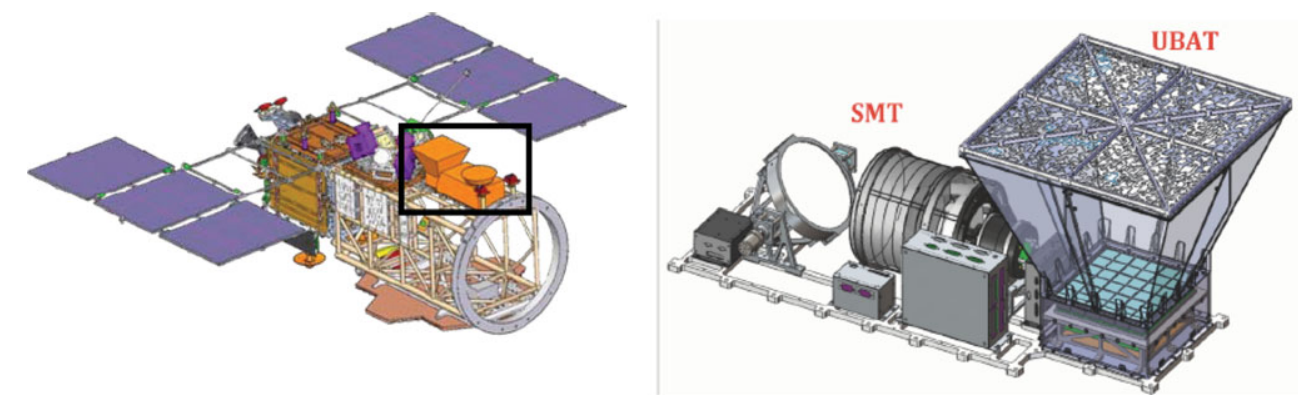

Figure 6. The integrated UFFO-pathfinder, to be launched aboard the Lomonosov spacecraft. The SMT enclosure is not shown in this drawing. Adapted from Chen et al. (2011).

of $\sim 190 \mathrm{~cm}^{2}$ ), multi-anode photo multipliers, and analog and digital readout electronics (Park et al. 2009, Chen et al. 2011; see Fig. 6).

\section{Summary}

Afterglow emission can be detected in all the electromagnetic range, in all timescales from seconds to months (the later only in some cases). A variety of features can be studied by different techniques (photometry, spectroscopy, polarimetry) to gain insight into the underlying hypernovae and progenitors, environments, abundances, metallicities, host galaxies, etc. The overall observational efforts provide additional clues for a better understanding of the reverse and forward shock in the framework of the existing theoretical models, which should also accommodate the last observational results (not always easy).

In order to shed additional light in the field, technological developments are also required, e.g. new instrumentation at large facilities (like GTC) and at robotic telescope networks (like BOOTES). Future space missions, like Lomonosov/UFFO-p (to be launched in 2012) should be well suited for studying GRB emission at very early stages.

\section{Acknowledgements}

I appreciate the contribution over the years of the members of my ARAE Group (arae.iaa.es) as well as others belonging to the BOOTES Collaboration (bootes.iaa.es), UFFO-p Team and additional long-term collaborators worldwide. I also acknowledge support from CSIC, INTA, Junta de Andalucía and the Spanish Ministry of Science and Innovation through Project AYA 2009-14000-C03-01 (including FEDER funds).

\section{References}

Berger, E. et al. 2011, ApJ, 743, 204

Bloom, J. et al. 1999, Nat, 401, 453

Bremer, M. et al. 1998, A\&A, 332, L13

Castro-Tirado, A. J. 1994, Ph.D. Thesis, Copenhagen Univ.

Castro-Tirado, A. J \& Gorosabel, J. 1999, A\&AS, 138, 449

Castro-Tirado, A. J. et al. 1999, Sci, 283, 2069

Castro-Tirado, A. J. 2001, Fourth INTEGRAL Workshop: Exploring the Gamma-ray Universe, ESA-SP Conf. Proc., SP-459, p. 367

Castro-Tirado, A. J. et al. 2006, A\&A, 459, 763 
Castro-Tirado, A. J. et al. 2010, A\&SA, 517, A61

Castro-Tirado, A. J. et al. 2012a, $A \mathscr{E} A$, in press

Castro-Tirado, A. J. et al. 2012b, BASI, in press

Castro-Tirado, A. J. et al. 2012c, $A \mathscr{G} A$, in preparation

Cobb, B. et al. 2010, ApJ, 718, L150

Costa, E. et al. 1998, Nat, 387, 783

Chen, P. et al. 2011, Proc. of the 32nd International Conference on Cosmic Rays (ICRC), in press (arXiv1106.3929C)

Denisenko \& Terekhov 2007, GCNC, 6155

Galama, T. et al. 1998, Nat, 395, 670

Gomboc, A. et al. 2009, GRB: Sixth Huntsville Symposium, AIP Conf. Proc., 133, 145

Goodman, J. 1986, ApJ, 308, L47

Gorosabel, J. et al. 2004, A\&A, 422, 113

Gorosabel, J. et al. 2006, A\&A, 459, L33

Gorosabel, J. et al. 2010, A\&A A, 522, A14

Greiner, J. et al. 2003, Nat, 426, 157

Guziy, S. et al. 2012, A\&A A, in preparation

Hjorth, J. et al. 2003, Nat, 423, 847

Hurley, K. et al. 2000, ApJS, 128, 549

Iwamoto, K. et al. 1998, Nat, 395, 672

Jelinek, M. et al. 2006, A\&\&A, 454, L119

Jelinek, M. et al. 2012, $A \mathscr{E} A$, in preparation

Kasen, X. et al. 2003, ApJ, 593, 788

Kulkarni, S. R. et al. 1999, Nat, 398, 389

Lazzati, D. et al. 2004, A\&AA, 422, 121

Meszaros, P. \& Rees, M. J. 1997, ApJ, 428, L29

Molinari, E. et al. 2007, ApJ, 469, L13

Nousek, J. A. et al. 2005, ApJ, 642, 389

Paczynski 1986, ApJ, 494, L45

Paczynski, B. \& Rhoads, J. E. 1986, ApJ, 418, L5

Panaitescu, A., Meszaros, P.\& Rees, M. J. 1998, ApJ, 503, 314

Pandey, S. B. 2010, ApJ, 714, 799

Park, I. et al. 2009, unpublished (arXiv0912.0773)

Pian, E. et al. 2000, ApJ, 536, 778

Sánchez-Ramírez, R. et al. 2012, A\&A, in preparation

Sari, R., Piran, T., \& Narayan, R. 1998, ApJ, 497, L17

Sokolov, V. V. et al. 2003, BSAO, 56, 5 [Stanek et al. 2003]Stanek03 Stanek, K. et al. 2003, ApJ, 591, L17

Starling, R. L. et al. 2005, MNRAS, 360, 305

Steele, I. et al. 2009, Nat, 462, 767

Tello-Salas, J. C. et al. 2012, A\&A, in preparation

Kasen, X. et al. 1993, Pisma Astron. Zh., 19, 686

de Ugarte Postigo, A. et al. 2005, A\&A, 443, 841

de Ugarte Postigo, A. et al. 2012, GCNC, 12802

Vergani, S. et al. 2011a, A\&A, 535, A127

Vergani, S. et al. 2011b, AN, 332, 292

Zhang, B., Kobayashi, S., \& Meszaros, P. 1993, ApJ, 595, 950

\section{Discussion}

L. Amatтi: What about the UFFO Mission? 
A. J. Castro-Tirado: The UFFO-p is scheduled for launch this year, aboard the Lomonosov spacecraft, in a low-Earth orbit. See also the contribution by H. Lim et al. (in these proceedings).

B. ZHANG: Can you say a few words about using ALMA for studying GRB afterglows?

A. J. Castro-Tirado: ALMA, thanks to its superb sensitivity, will opening a new era in the study of GRB afterglows detecting most of them if observed closed to the passage of the peak synchrotron emission. See also the contribution by A. de Ugarte Postigo et al. (in these proceedings). 